Dass eine moderne Immuntherapie nicht nur bei akuter lymphatischer Leukämie (ALL) erfolgreich sein kann, machte Philippe Armand, Boston, MA/ USA, unter anderem am Beispiel des diffusen, großzelligen B-Zell- (DLBCL) oder follikulären Lymphoms deutlich. In der CTL019-Studie wurden 26 Patienten mit autologen T-Lymphozyten behandelt, deren T-Zell-Rezeptor gentechnisch verändert worden war, sodass sie zu CAR-T-Zellen wurden. 15 Patienten hatten ein DLBCL und waren bereits dreifach erfolglos vorbehandelt worden. Die Gesamtansprechrate habe nach drei Monaten bei $47 \%$ gelegen, so Armand. Der Anteil der Patienten mit einem
CR habe $40 \%$ betragen. Als CR ist bei DLBCL das Fehlen jeglicher residueller Raumforderung definiert.

Ein anderes Beispiel: die Ergebnisse von aktuellen Studien mit Hodgkin-Lymphom-Patienten, die mit PD-1-Checkpointhemmern behandelt wurden. So sei in einer Phase-I-Studie mit 54 Patienten, die zuvor median bis zu fünf Therapien erhalten hatten, ein Tumoransprechen von $94 \%$ erzielt worden, sagte Armand. Und in einer Phase-II-Studie mit 170 Patienten habe eine Gesamtansprechrate von $65-80 \%$ sowie eine CR-Rate von 10-30 \% erzielt werden können, also deutlich bessere Ergebnisse als nach einer konventionellen Chemotherapie. Weitere
Studien hätten gezeigt, dass nach einer Immuntherapie mit CAR-T-Zellen oder Checkpointhemmern eine allo-SCT möglich sei. So gesehen könne die Immuntherapie als Vorbereitung oder zum Verschieben der Transplantation genutzt werden. Am besten solle die Immuntherapie frühzeitig erfolgen.

Letztlich sei der Erfolg nach einer Immuntherapie, gemessen am Parameter PFS, ähnlich gut wie nach einer alloSCT. Allerdings handele es sich bei Therapieversagen nach Immuntherapie um Rezidive, nach einer SCT dagegen teilweise um Folgen der Abstoßungsreaktionen. Im Gegensatz zur allo-SCT bei Lymphomen sei die Immuntherapie der-

\title{
Nachgefragt
}

\section{Immuntherapie bei ALL mit relevantem Überlebensvorteil}

Bei Erwachsenen mit akuter lymphatischer Leukämie (ALL) ist die Prognose noch immer deutlich schlechter als bei Kindern. Das Antikörperkonstrukt Blinatumomab kann diese deutlich verbessern. Springer Medizin sprach mit Prof. Max S. Topp, Würzburg, über die ersten Ergebnisse der TOWER-Studie.

? Die TOWER-Studie wurde vorzeitig abgebrochen, warum?

Prof. Max S. Topp: In der TOWER-Studie wurde untersucht, ob mit Blinatumomab ein verlängertes Überleben (OS) bei einer rezidivierten oder refraktären $\mathrm{ALL}$ erreicht werden kann. Der Vergleichsarm war eine Standardchemotherapie [Topp MS et al. EHA. 2016;Abstr S149]. Die Studie wurde abgebrochen, weil in der vordefinierten Interimsanalyse ein klares Signal gesehen worden ist, dass in der Kohorte Blinatu-

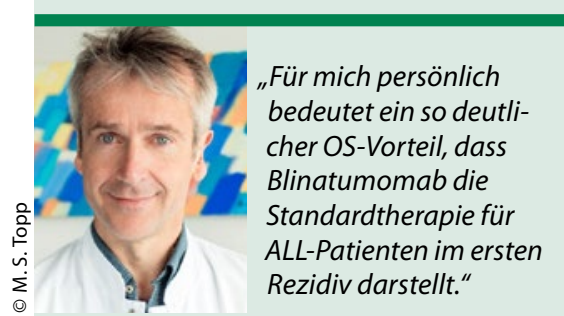

Prof. Dr. Max S. Topp

Medizinische Klinik und Poliklinik II Universitätsklinikum Würzburg momab in Bezug auf das OS der Standardchemotherapie überlegen ist.

? War das ein relevanter OS-Vorteil? Topp: Für Patienten, die ein Rezidiv einer ALL haben, liegt das mediane OS in der Literatur bei knapp vier Monaten. Das wurde auch in dieser Studie bestätigt: Im Chemotherapie-Arm überlebten sie im Median etwa vier Monate. Bei mit Blinatumomab behandelten Patienten wurde das OS fast verdoppelt - auf 7,7 Monate. Das ist wirklich relevant für diese Patienten!

\section{? Wie sicher ist Blinatumomab?}

Topp: In der Studie war bei Therapie mit Blinatumomab eine Reduktion der für Patienten relevanten Grad-3/4-Ereignisse bezüglich der infektiösen Komplikationen und der schweren Hämatotoxizitäten gegenüber der Chemotherapie zu sehen. Das führte in der Regel dazu, dass wir die Patienten frühzeitig aus dem Krankenhaus entlassen konnten. Überraschend war, dass die für Blinatumomab beschriebene
Neurotoxizität, die in der Phase-Illb-Studie noch bei $14-15 \%$ in einem Grad 3/4 auftrat, in der Tower-Studie nur bei $8 \%$ beobachtet wurde. Das liegt wahrscheinlich daran, dass man mehr Erfahrung gesammelt hat, wie man damit umgehen kann.

Blinatumomab kann außerdem ein Zytokin-Release-Syndrom auslösen. Die Rate schwerer Zytokin-Release-Syndrome liegt erfreulicherweise deutlich unter $10 \%$, wir reden jetzt über 2-3\%. Das ist sehr gut beherrschbar, weil ein Zytokin-Release-Syndrom berechenbar in den ersten zwei bis drei Tagen der Therapie auftritt und weil Blinatumomab eine kurze Halbwertszeit hat.

? Welchen Einfluss haben diese Ergebnisse für die Rezidiv-Therapie der ALL in der Praxis?

Topp: Für mich persönlich bedeutet ein so deutlicher OS-Vorteil in einer randomisierten Phase-III-Studie, dass Blinatumomab die Standardtherapie für Patienten im ersten Rezidiv bei einer ALL darstellt. Das gilt vor allem, weil für die Studie besonders schwer behandelbare Patienten ausgewählt wurden, etwa Patienten, die nach Stammzelltransplantation ein Rezidiv erlitten hatten.

Das vollständige Interview lesen Sie hier: wWw.springermedizin.de/link/10627898 